\title{
PERTURBATION THEORY AND CONDENSATES
}

\author{
G. GRUNBERG* \\ Centre de Physique Théorique de l'Ecole Polytechnique \\ 91128 Palaiseau Cedex - France. \\ CNRS UPRA 0014
}

\begin{abstract}
A convergent scheme to deal with perturbation theory in presence of infrared renormalons is presented, extending its validity to large orders and stronger coupling. An unambiguous meaning is given to the condensates appearing in the operator product expansion. The QCD sum rule approach is justified, but definite corrections are pointed out.
\end{abstract}

* email : PTHGRU @ FRPOLY11.POLYTECHNIQUE.FR 
1. A standard tool to introduce non-perturbative effects starting from weak coupling is the operator product expansion (OPE), developped in ref.[1] in the "QCD sum rule" approach. However, the condensates in the OPE seem to some extent ambiguous [2], and related to the resummation prescription used for the perturbative part (plagued with the renormalon [3] problem), so that the validity of the procedure of ref.[1] is not apparent. The aim of the present note is to provide the necessary clarification. A practical procedure will be given to handle the divergent perturbation theory in presence of renormalons, extending its usefulness towards large orders and strong coupling (provided the OPE still converges). Furthermore, the condensates introduced in ref.[1] will be shown to have a perfectly unambiguous meaning (although compatible with the finding of ref.[2]), thus justifying to some extent the QCD sum rule approach. Some definite corrections to the framework of ref.[1] will nevertheless emerge.

\section{Consider a generic correlator (I drop Lorentz indices) :}

$\pi(Q)=\int d^{4} x \exp ($ iqx $)<0|T(j(x) j(0))| 0>$, and the associated renormalization group (RG) invariant quantity $R(Q)=\frac{d \pi}{d \ln Q^{2}}-\left.\frac{d \pi}{d \ln Q^{2}}\right|_{\alpha=0}$. The OPE yields the representation :

$$
\mathrm{R}(\mathrm{Q})=\mathrm{R}_{\mathrm{PT}}(\alpha)+\mathrm{G}_{0}(\alpha)+\ldots
$$

where $\alpha=\alpha(\mu)$ is the coupling at scale $\mu, \mathrm{R}_{\mathrm{PT}}(\alpha)=\sum_{n=0}^{\infty} \mathrm{r}_{\mathrm{n}} \alpha^{\mathrm{n}+1}$ the perturbative contribution, $\mathrm{G}_{0}(\alpha)$ the leading condensate contribution, and the dots denote the contribution of higher dimensional condensates. For instance, if $\mathrm{G}_{0}(\alpha)$ is the gluon condensate contribution, $\mathrm{G}_{0}(\alpha)=1 / \mathrm{Q}^{4}\left\langle 0\left|\mathrm{~F}^{2}\right| 0\right\rangle_{\mu} \mho(\mathrm{Q} / \mu, \alpha)$, where $\mho(\mathrm{Q} / \mu, \alpha)$ is the corresponding coefficient function. Using the RG (note that $R_{\mathrm{PT}}(\alpha)$ and $\mathrm{G}_{0}(\alpha)$ are separately RG invariant) one easily finds the $\alpha$ dependence of $G_{0}(\alpha)$, for a condensate of dimension $d$ :

$$
G_{0}(\alpha)=C\left(\frac{\mu^{2}}{Q^{2}}\right)^{d / 2} \exp \left(d / 2 \beta_{0} \alpha\right) \alpha^{\delta}[1+O(\alpha)]
$$

where $\delta=\frac{d}{2} \frac{\beta_{1}}{\beta_{0}^{2}}-\frac{\gamma_{0}}{\beta_{0}}, \beta_{0}$ and $\beta_{1}$ are the $\beta$-function coefficients : $\frac{d \alpha}{d \ln \mu^{2}}=\beta_{0} \alpha^{2}+\beta_{1} \alpha^{3}+\ldots, \gamma_{0}$ is the one loop anomalous dimension of the corresponding operator, and $\mathrm{C}$ a scale-independent constant which contains the truly non-perturbative information. Eq.(2) displays for $\alpha \rightarrow 0$ an essential singularity, so that the OPE acquires an unambiguous meaning only in presence of a resummation prescription for $\mathrm{R}_{\mathrm{PT}}(\alpha)$. Following ref.[2], I assume the latter is 
provided by Borel resummation above or under the positive real axis in the Borel plane, which leads to a unique analytic function $\mathrm{R}_{\mathrm{PT}}(\alpha)$ (such that $\mathrm{R}_{\mathrm{PT}}(\alpha)$ has no essential singularity on the first sheet [4] for $\alpha \rightarrow 0$ ), albeit one with a cut for $\alpha>0$ in presence of renormalon singularities. Of course, once this "canonical" prescription is established, it is always possible to shift exponentially small terms between $\mathrm{R}_{\mathrm{PT}}(\alpha)$ and $\mathrm{G}_{0}(\alpha)$, and this will be done in fact below (see eq.(16)).

3. Consider the Borel representation :

$$
\mathrm{R}_{\mathrm{PT}}(\alpha)=\int_{0}^{\infty} \mathrm{dz} \exp (-\mathrm{z} / \alpha) \mathrm{R}_{\mathrm{PT}}(\mathrm{z})
$$

The Borel transform series $\mathrm{R}_{\mathrm{PT}}(\mathrm{z})=\sum_{\mathrm{n}=0}^{\infty} \frac{\mathrm{r}_{\mathrm{n}}}{\mathrm{n} !} \mathrm{z}^{\mathrm{n}}$ are believed to have a finite convergence radius. Call $\mathrm{z}_{0}$ the first singularity of $\mathrm{R}_{\mathrm{PT}}(\mathrm{z})$ on the positive real axis. This is usually the first infrared (IR) renormalon, arising from "IR Landau ghost" effects [3,5,6,7]. Let us assume (see below) :

$$
\mathrm{R}_{\mathrm{PT}}(\mathrm{z}) \underset{\mathrm{z} \rightarrow \mathrm{z}_{0}}{\sim} \mathrm{R}_{\mathrm{PT}, 0}(\mathrm{z}) \equiv \mathrm{K}\left(\frac{\mu^{2}}{\mathrm{Q}^{2}}\right)^{-\mathrm{z}_{0} \beta_{0}} \frac{1}{\left(1-\frac{\mathrm{z}}{\mathrm{z}_{0}}\right)^{\gamma}}\left[1+\mathrm{O}\left(1-\frac{\mathrm{z}}{\mathrm{z}_{0}}\right)\right]
$$

where $\mathrm{K}$ is a scale independent factor, which yields the large $\mathrm{n}$ behavior (barring ultraviolet (UV) renormalons at $\mathrm{z}<0$ ) :

with

$$
r_{n} \sim K\left(\frac{\mu^{2}}{Q^{2}}\right)^{-z_{0} \beta_{0}} f_{n}
$$

$$
f_{n} \equiv \frac{1}{z_{0}^{n}} \frac{\Gamma(n+\gamma)}{\Gamma(\gamma)}\left[1+O\left(\frac{1}{n}\right)\right]
$$

Eq.(4) implies the corresponding "renormalon contribution" to $\mathrm{R}_{\mathrm{PT}}(\alpha)$ :

$$
\mathrm{R}_{\mathrm{PT}, 0}(\alpha)=\int_{0}^{\infty} \mathrm{dz} \exp (-\mathrm{z} / \alpha) \mathrm{R}_{\mathrm{PT}, 0}(\mathrm{z})
$$

acquires an imaginary part for $\alpha>0$ : 


$$
\begin{aligned}
\operatorname{Im} R_{\mathrm{PT}, 0}(\alpha) & =\int_{\mathrm{z}_{0}}^{\infty} \mathrm{dz} \exp (-\mathrm{z} / \alpha) \operatorname{Im} \mathrm{R}_{\mathrm{PT}, 0}(\mathrm{z}) \\
& = \pm \mathrm{Kz} \mathrm{z}_{0}^{\gamma} \Gamma(1-\gamma) \sin (\pi(1-\gamma))\left(\frac{\mu^{2}}{\mathrm{Q}^{2}}\right)^{-\mathrm{z}_{0} \beta_{0}} \exp \left(-\mathrm{z}_{0} / \alpha\right) \alpha^{1-\gamma}[1+\mathrm{O}(\alpha)]
\end{aligned}
$$

(I assume momentarily that $\gamma<1$, so that the integral in eq.(7) converges at $\mathrm{z}=\mathrm{z}_{0}$; however, the result for $\operatorname{Im} R_{\mathrm{PT}, 0}(\alpha)$ is of general validity). The sign in eq.(7) depends whether one integrates above or under the real axis in eq.(6) : the ambiguity in the choice of contour thus leads to one and the same analytic function, but on different sides of its cut, for $\alpha>0$. In any case, the resummed $\mathrm{R}_{\mathrm{PT}}(\alpha)$ cannot give the full answer for $\mathrm{R}$, and consistency of QCD requires the pathologies in $R_{\mathrm{PT}}(\alpha)$ to be cured $[2,6]$ by taking into account some non-perturbative information, provided by the vacuum condensates in the OPE. One indeed assumes the constant $C$ in eq.(2) is actually complex, with an ambiguous imaginary part : $C=C_{R} \pm i C_{I}$, which cancells the one coming from Borel resummation (this imaginary part is related [2] to the appearance of non-logarithmic UV divergences in the corresponding condensate). Comparing eq.(7) with eq.(2), and requiring cancellation, gives the relations :

$$
\begin{array}{r}
-z_{0} \beta_{0}=\frac{d}{2} \\
1-\gamma=\delta
\end{array}
$$

which fix the position [5] and the power [6] of the singularity (the $O(\alpha)$ series in eq.(7) must similarly be identical [8] to those in eq.(2)).

4. Up to now, I have recalled essentially common wisdom. To clarify the occurence of an "ambiguous" imaginary part in the condensate contribution, and build up a practical resummation procedure, it is however necessary to give a more detailed picture of how the previous cancellation takes place, which makes use of the analyticity properties in the $\alpha$-plane. The main point is that, in addition to eq.(2), the $R G$ allows another solution $\widetilde{G}_{0}(\alpha)$, which differs from $G_{0}(\alpha)$ simply by changing $\alpha^{\delta}$ into $(-\alpha)^{\delta}$, leaving everything else (including the $\mathrm{O}(\alpha)$ series), apart from the overall normalization, the same :

$$
\widetilde{G}_{0}(\alpha)=\widetilde{C}\left(\frac{\mu^{2}}{Q^{2}}\right)^{d / 2} \exp \left(d / 2 \beta_{0} \alpha\right)(-\alpha)^{\delta}[1+O(\alpha)]
$$


If $\delta \neq$ integer (the generic case), $\widetilde{\mathrm{G}}_{0}(\alpha)$ has a cut and becomes imaginary for $\alpha>0$, and differs from $\mathrm{G}_{0}(\alpha)$ only by the (double-valued) normalization factor $\widetilde{\mathrm{C}} \exp ( \pm \mathrm{i} \pi \delta)$. The general solution of the RG equation for the condensate contribution is therefore the $\operatorname{sum}^{1}$ of eq.(2) and (9), with C, $\widetilde{\mathrm{C}}$ real, and eq.(1) becomes :

$$
\mathrm{R}(\mathrm{Q})=\mathrm{R}_{\mathrm{PT}}(\alpha)+\left[\mathrm{G}_{0}(\alpha)+\widetilde{\mathrm{G}}_{0}(\alpha)\right]+\ldots
$$

Consequently, for $\alpha>0 \mathrm{C}$ is replaced by $\mathrm{C}+\widetilde{\mathrm{C}} \exp ( \pm \mathrm{i} \pi \delta) \equiv \mathrm{C}_{\mathrm{R}} \pm \mathrm{iC} \mathrm{C}_{\mathrm{I}}$. Consistency now requires $\widetilde{G}_{0}(\alpha)$ cancells a similar piece in the renormalon contribution $R_{P T, 0}(\alpha)$ (eq.(6)). This is most clearly seen by introducing the Borel representation of $\widetilde{\mathrm{G}}_{0}(\alpha)$ following from eq.(9) :

$$
\widetilde{\mathrm{G}}_{0}(\alpha)=\int_{\mathrm{z}_{0}}^{\infty} \mathrm{dz} \exp (-\mathrm{z} / \alpha) \widetilde{\mathrm{G}}_{0}(\mathrm{z})
$$

with

$$
\widetilde{G}_{0}(\mathrm{z})=-\widetilde{\mathrm{C}} \frac{\mathrm{z}_{0}^{\delta-1}}{\Gamma(\delta)}\left(\frac{\mu^{2}}{\mathrm{Q}^{2}}\right)^{\mathrm{d} / 2} \frac{1}{\left(1-\frac{\mathrm{z}}{\mathrm{z}_{0}}\right)^{1-\delta}}\left[1+\mathrm{O}\left(1-\frac{\mathrm{z}}{\mathrm{z}_{0}}\right)\right]
$$

where the lower bound of integration is determined by the relation (eq.(8)) $z_{0}=-d / 2 \beta_{0}$, and I assume momentarily $\delta>0$ for convergence at $z=z_{0}$. For future use, $I$ note the similar Borel representation of $\mathrm{G}_{0}(\alpha)$ :

$$
\mathrm{G}_{0}(\alpha)=\int_{\mathrm{z}_{0}}^{\infty} \mathrm{dz} \exp (-\mathrm{z} / \alpha) \mathrm{G}_{0}(\mathrm{z})
$$

with

$$
G_{0}(z)=C \frac{z_{0}^{\delta-1}}{\Gamma(\delta)}\left(\frac{\mu^{2}}{Q^{2}}\right)^{d / 2} \frac{1}{\left(\frac{z}{z_{0}}-1\right)^{1-\delta}}\left[1+O\left(1-\frac{z}{z_{0}}\right)\right]
$$

(the $O\left(1-\frac{\mathrm{Z}}{\mathrm{Z}_{0}}\right)$ series in eq.(11) and (12) are the same). The condition that the imaginary parts of $\mathrm{R}_{\mathrm{PT}, 0}(\alpha)$ and $\widetilde{\mathrm{G}}_{0}(\alpha)$ cancell is equivalent to the identity :

$$
\mathrm{R}_{\mathrm{PT}, 0}(\mathrm{z}) \equiv-\widetilde{\mathrm{G}}_{0}(\mathrm{z})
$$

1 There is a parallel "two-component" representation for the condensate itself :

$$
\begin{aligned}
<0|O| 0>_{\mu} & =\mathrm{C}\left(\mu^{2}\right)^{\mathrm{d} / 2} \exp \left(\mathrm{d} / 2 \beta_{0} \alpha\right)(\alpha)^{\delta}[1+\mathrm{O}(\alpha)] \\
& +\widetilde{\mathrm{C}}\left(\mu^{2}\right)^{\mathrm{d} / 2} \exp \left(\mathrm{d} / 2 \beta_{0} \alpha\right)(-\alpha)^{\delta}[1+\mathrm{O}(\alpha)]
\end{aligned}
$$


Comparing eq.(4) and (11) one indeed recovers eq.(8), and also :

$$
K=\widetilde{C} \frac{z_{0}^{\delta-1}}{\Gamma(\delta)}
$$

i.e. the renormalon residue $\mathrm{K}$ is related to the condensate parameter $\widetilde{\mathrm{C}}$, and is therefore expected to be a process independent quantity. Eq.(14) is valid even if $\delta<0$. In particular, for finite $\widetilde{\mathrm{C}}, \mathrm{K}$ vanishes when $\delta=0,-1,-2$, etc... (i.e. $\gamma=1,2,3$, etc...), which means there is then no renormalon at $\mathrm{z}=\mathrm{z}_{0}$, as expected since for these values $\widetilde{\mathrm{G}}_{0}(\alpha)$ is real for $\alpha$ real. Conversely, $\widetilde{\mathrm{C}}$ is determined by information contained in the perturbative series (although taken to all orders [7]!), since eq.(13) gives :

$$
\widetilde{\mathrm{G}}_{0}(\alpha)=-\int_{\mathrm{z}_{0}}^{\infty} \mathrm{dz} \exp (-\mathrm{z} / \alpha) \mathrm{R}_{\mathrm{PT}, 0}(\mathrm{z})
$$

Eq.(15) suggests to refer to $\widetilde{\mathrm{G}}_{0}(\alpha)$ as the "perturbation theory determined ${ }^{2}$ part" of the condensate contribution.

5. A convergent scheme for perturbation theory emerges from the previous considerations. Let us define a "regularized perturbative part" by :

$$
\begin{aligned}
\mathrm{R}_{\mathrm{PT}}^{\mathrm{reg}}(\alpha) & =\mathrm{R}_{\mathrm{PT}}(\alpha)+\widetilde{\mathrm{G}}_{0}(\alpha) \\
& =\int_{0}^{\infty} \mathrm{dz} \exp (-\mathrm{z} / \alpha) \mathrm{R}_{\mathrm{PT}}(\mathrm{z})+\int_{\mathrm{z}_{0}}^{\infty} \mathrm{dz} \exp (-\mathrm{z} / \alpha) \widetilde{\mathrm{G}}_{0}(\mathrm{z})
\end{aligned}
$$

so that the total $\mathrm{R}$ is given from eq.(10) by :

$2 \widetilde{\mathrm{G}}_{0}(\alpha)$ is different from - R $\mathrm{PT}, 0(\alpha)$ (eq.(6)). The latter is sometimes identified [9] to a "perturbative condensate" contribution, but the definition eq.(15) is preferable, which yields a RG invariant quantity having the correct $\exp \left(-\mathrm{z}_{0} / \alpha\right)$ behavior, both properties not shared by $\mathrm{R}_{\mathrm{PT}, 0}(\alpha)$. The difference is very simple in the coupling constant dispersion representation of the renormalon, since the leading term in $\widetilde{\mathrm{G}}_{0}(\alpha)$ can be written as (for $0<\delta<1$ ):

$$
\widetilde{\mathrm{G}}_{0}(\alpha)=\widetilde{\mathrm{C}}\left(\frac{\mu^{2}}{\mathrm{Q}^{2}}\right)^{\mathrm{d} / 2} \exp \left(-\mathrm{z}_{0} / \alpha\right) \frac{\alpha}{\pi} \int_{0}^{\infty} \frac{\mathrm{d} \alpha^{\prime}}{\alpha^{\prime}} \frac{\sin (\pi \delta) \alpha^{\prime \delta}}{\alpha^{\prime}-\alpha}
$$

which differs from the corresponding representation [9] of $-\mathrm{R}_{\mathrm{PT}, 0}(\alpha)$ only by the factor $\exp \left(-\mathrm{z}_{0} / \alpha\right)$, which is pushed outside the dispersion integral. 


$$
R(Q)=R_{P T}^{r e g}(\alpha)+G_{0}(\alpha)+\ldots
$$

i.e. the sum $^{3}$ of the "regularized perturbative part" and the "genuine" non-perturbative condensate contribution $\mathrm{G}_{0}(\alpha)$. Note that $\mathrm{R}_{\mathrm{PT}}^{\text {reg }}(\alpha)$ is now real and non-ambiguous for $\alpha>0$. Eq.(16) allows to devise a convergent approximation scheme for $\mathrm{R}_{\mathrm{PT}}^{\mathrm{reg}}(\alpha)$. Consider the series :

$$
\mathrm{R}_{\mathrm{PT}}^{\mathrm{reg}(\mathrm{N})}(\alpha)=\mathrm{R}_{\mathrm{PT}}^{(\mathrm{N})}(\alpha)+\widetilde{\mathrm{G}}_{0}^{(\mathrm{N})}(\alpha)
$$

where

$$
\mathrm{R}_{\mathrm{PT}}^{(\mathrm{N})}(\alpha)=\int_{0}^{\infty} \mathrm{dz} \exp (-\mathrm{z} / \alpha) \mathrm{R}_{\mathrm{PT}}^{(\mathrm{N})}(\mathrm{z})=\sum_{\mathrm{n}=0}^{\mathrm{N}} \mathrm{r}_{\mathrm{n}} \alpha^{\mathrm{n}+1}
$$

with

$$
R_{\mathrm{PT}}^{(\mathrm{N})}(\mathrm{z})=\sum_{\mathrm{n}=0}^{\mathrm{N}} \frac{\mathrm{r}_{\mathrm{n}}}{\mathrm{n} !} \mathrm{z}^{\mathrm{n}}
$$

is standard perturbation theory, and :

$$
\widetilde{G}_{0}^{(N)}(\alpha)=\int_{z_{0}}^{\infty} d z \exp (-z / \alpha) \widetilde{G}_{0}^{(N)}(z)=\exp \left(-z_{0} / \alpha\right) \sum_{n=0}^{N} \frac{d^{n} \widetilde{G}_{0}^{(N)}}{d z^{n}}\left(z=z_{0}\right) \times \alpha^{n+1}
$$

where

$$
\widetilde{G}_{0}^{(N)}(z)=-K\left(\frac{\mu^{2}}{Q^{2}}\right)^{-z_{0} \beta_{0}} \sum_{n=0}^{N} \frac{f_{n}}{n !} z^{n}
$$

are the $\mathbf{z} \rightarrow \mathbf{0}$ series of $\widetilde{\mathrm{G}}_{0}(\mathrm{z})$ trunkated at order $\mathrm{N}$, hence :

$$
\frac{\mathrm{d}^{\mathrm{n}} \widetilde{\mathrm{G}}_{0}^{(\mathrm{N})}}{\mathrm{dz^{n }}}\left(\mathrm{z}=\mathrm{z}_{0}\right)=-\mathrm{K}\left(\frac{\mu^{2}}{\mathrm{Q}^{2}}\right)^{-\mathrm{z}_{0} \beta_{0}} \sum_{\mathrm{p}=\mathrm{n}}^{\mathrm{N}} \frac{\mathrm{f}_{\mathrm{p}}}{(\mathrm{p}-\mathrm{n}) !} \mathrm{z}_{0}^{\mathrm{p}-\mathrm{n}}
$$

(eq.(20) is obtained most easily by reexpanding $\widetilde{\mathrm{G}}_{0}^{(\mathrm{N})}(\mathrm{z})$ around $\mathrm{z}=\mathrm{z}_{0}$ ). Ignoring the extra IR renormalons at $z>z_{0}$ (also expected in $\widetilde{G}_{0}(z)$ ), the $f_{n}$ 's are obtained as convergent series (eq.(5)) from the $\mathrm{z} \rightarrow \mathrm{z}_{0}$ expansion of $\widetilde{\mathrm{G}}_{0}(\mathrm{z})=-\mathrm{R}_{\mathrm{PT}, 0}(\mathrm{z})$ (eq.(4),(11)), since $\mathrm{z}=0$ is within the circle of convergence of the $O\left(1-\frac{z}{z_{0}}\right)$ series $^{4}$.In the absence of $U V$ renormalon ${ }^{5}$ at $z=1 / \beta_{0}<0$, the

\footnotetext{
${ }^{3} \mathrm{Eq} .(16)$ and (17) are similar in spirit to, but different in content from, a procedure suggested in ref. [10].
} 
series eq.(18) are expected to converge (at least for $\alpha$ not too large), since the large $\mathrm{N}$ divergencies of the $\mathrm{R}_{\mathrm{PT}}^{(\mathrm{N})}(\alpha)$ and of the $\widetilde{\mathrm{G}}_{0}^{(\mathrm{N})}(\alpha)$ series should cancell. A heuristic argument for the convergence is as follows : imagine replacing $\widetilde{\mathrm{G}}_{0}(\mathrm{z})$ in eq.(16) by $-\mathrm{R}_{\mathrm{PT}}(\mathrm{z})$, which turns $\mathrm{R}_{\mathrm{PT}}^{\text {reg }}(\alpha)$ into :

$$
\overline{\mathrm{R}}_{\mathrm{PT}}(\alpha)=\int_{0}^{\mathrm{z}_{0}} \mathrm{dz} \exp (-\mathrm{z} / \alpha) \mathrm{R}_{\mathrm{PT}}(\mathrm{z})
$$

In the absence of UV renormalons, the convergence properties of the $\mathrm{R}_{\mathrm{PT}}^{\mathrm{reg}(\mathrm{N})}(\alpha)$ series and of the series $\overline{\mathrm{R}}_{\mathrm{PT}}^{(\mathrm{N})}(\alpha)=\int_{0}^{\mathrm{z}_{0}} \mathrm{dz} \exp (-\mathrm{z} / \alpha) \mathrm{R}_{\mathrm{PT}}^{(\mathrm{N})}(\mathrm{z})$ should be the same. Indeed we have :

$$
\begin{aligned}
\overline{\mathrm{R}}_{\mathrm{PT}}^{(\mathrm{N})}(\alpha) & =\int_{0}^{\infty} \mathrm{d} z \exp (-\mathrm{z} / \alpha) \mathrm{R}_{\mathrm{PT}}^{(\mathrm{N})}(\mathrm{z})-\int_{\mathrm{z}_{0}}^{\infty} \mathrm{dz} \exp (-\mathrm{z} / \alpha) \mathrm{R}_{\mathrm{PT}}^{(\mathrm{N})}(\mathrm{z}) \\
& =\sum_{\mathrm{n}=0}^{\mathrm{N}} \mathrm{r}_{\mathrm{n}} \alpha^{\mathrm{n}+1}-\exp \left(-\mathrm{z}_{0} / \alpha\right) \sum_{\mathrm{n}=0}^{\mathrm{N}} \frac{\mathrm{d}^{\mathrm{n}} \mathrm{R}_{\mathrm{PT}}^{(\mathrm{N})}}{\mathrm{dz}}\left(\mathrm{z}=\mathrm{z}_{0}\right) \times \alpha^{\mathrm{n}+1}
\end{aligned}
$$

with

$$
\frac{d^{n} R_{P T}^{(N)}}{d z^{n}}\left(z=z_{0}\right)=\sum_{p=n}^{N} \frac{r_{p}}{(p-n) !} z_{0}^{p-n}
$$

But the large $n$ behavior of the $r_{n}$ 's is given by eq. (5), so that $-\frac{\mathrm{d}^{\mathrm{n}} \mathrm{R}_{\mathrm{PT}}^{(\mathrm{N})}}{\mathrm{dz}^{\mathrm{n}}}\left(\mathrm{z}=\mathrm{z}_{0}\right)$ becomes $\frac{\mathrm{d}^{\mathrm{n}} \widetilde{\mathrm{G}}_{0}^{(\mathrm{N})}}{\mathrm{dz}^{\mathrm{n}}}\left(\mathrm{z}=\mathrm{z}_{0}\right)$ (eq.(22)) for large $\mathrm{n}$. On the other hand, the $\overline{\mathrm{R}}_{\mathrm{PT}}^{(\mathrm{N})}(\alpha)$ series are convergent [5] (if the singularity of $\mathrm{R}_{\mathrm{PT}}(\mathrm{z})$ at $\mathrm{z}=\mathrm{z}_{0}$ is integrable, i.e. if $\delta>0$ ) in absence of $\mathrm{UV}$ renormalon, since the range of integration of eq.(23) is within the circle of convergence of the $\mathrm{R}_{\mathrm{PT}}^{(\mathrm{N})}(\mathrm{z})$ series (a more complete argument can be given in term of the $\Delta_{0}(z)$ function introduced below).

\footnotetext{
${ }^{4}$ The IR renormalon at $z=z_{1}>z_{0}$ may require an analytic continuation to $z=0$ : one needs the exact $f_{n}$ 's, not only their large $n$ asymptotics.

5 The UV renormalon is disposed of by the standard trick $[5,10,11]$ of a conformal mapping $z \rightarrow u(z)=\frac{\sqrt{1-z \beta_{0}}-1}{\sqrt{1-z \beta_{0}}+1}, z(u)=-\frac{4 u}{\beta_{0}(1-u)^{2}}$. One then replaces in the Borel integrals the trunkated series in power of $z$ by trunkated series in power of $u$, i.e. $R_{P T}^{(\mathrm{N})}(\mathrm{z}) \rightarrow \mathbf{R}_{\mathrm{PT}}^{(\mathrm{N})}[\mathrm{u}(\mathrm{z})]$, where $\mathrm{R}_{\mathrm{PT}}(\mathrm{u}) \equiv \mathrm{R}_{\mathrm{PT}}[\mathrm{z}(\mathrm{u})]$, and similarly $\widetilde{\mathrm{G}}_{0}^{(\mathrm{N})}(\mathrm{z}) \rightarrow \widetilde{\mathrm{G}}_{0}^{(\mathrm{N})}[\mathrm{u}(\mathrm{z})]$.
} 
Adding the "genuine" condensate contribution $\mathrm{G}_{0}(\alpha)$, one thus gets the convergent approximation to $\mathrm{R}$ :

$$
\mathrm{R}(\mathrm{Q}) \simeq \mathrm{R}^{(\mathrm{N})}(\alpha) \equiv \mathrm{R}_{\mathrm{PT}}^{(\mathrm{N})}(\alpha)+\left[\widetilde{\mathrm{G}}_{0}^{(\mathrm{N})}(\alpha)+\mathrm{G}_{0}(\alpha)\right]+\ldots
$$

(where $G_{0}(\alpha)$ is taken $N$-independent, since it is given by the convergent series eq.(2) in absence of $z=z_{1}$ renormalon). Eq.(26) is exactly the standard form of OPE used in ref.[1], except for the correction term $\widetilde{\mathrm{G}}_{0}^{(\mathrm{N})}(\alpha)$, which may be important when $\mathrm{N}$ or $\alpha$ are large, and takes care of the IR renormalon. Ref.[1] effectively makes the assumption that this term can be ignored ; its actual magnitude should be extracted from experiment, since the renormalon residue $\mathrm{K}$, or $\widetilde{\mathrm{C}}$ (the only unknown "all order" parameter in eq.(21)) is rather difficult to compute [7]. Taking it into account will affect the value of the "genuine" condensate parameter $C$. Note that $\widetilde{G}_{0}^{(\mathrm{N})}(\alpha)$ is an N-dependent quantity, which behaves as $\mathrm{O}\left(\alpha \exp \left(-\mathrm{z}_{0} / \alpha\right)\right)$ at small $\alpha$, whereas $\mathrm{G}_{0}(\alpha)$ is $\mathrm{O}\left(\alpha^{\delta} \exp \left(-\mathrm{z}_{0} / \alpha\right)\right)$. If $\delta$ is not very different from $1, \widetilde{\mathrm{G}}_{0}^{(\mathrm{N})}(\alpha)$ may be merged with $\mathrm{G}_{0}(\alpha)$ to yield an "effective" Ndependent $[10]$ condensate contribution.

Another useful expression for $\mathrm{R}_{\mathrm{PT}}^{\mathrm{reg}}(\alpha)$, which generalizes easily to the case of additional IR renormalons at $\mathrm{z}>\mathrm{z}_{0}$, may be given in term of $\overline{\mathrm{R}}_{\mathrm{PT}}(\alpha)$ (eq.(23)). One has :

$$
\mathrm{R}_{\mathrm{PT}}^{\mathrm{reg}}(\alpha)=\overline{\mathrm{R}}_{\mathrm{PT}}(\alpha)+\Delta_{0}(\alpha)
$$

where

$$
\Delta_{0}(\alpha)=\int_{\mathrm{z}_{0}}^{\infty} \mathrm{dz} \exp (-\mathrm{z} / \alpha) \Delta_{0}(\mathrm{z})
$$

with

$$
\Delta_{0}(\mathrm{z})=\mathrm{R}_{\mathrm{PT}}(\mathrm{z})+\widetilde{\mathrm{G}}_{0}(\mathrm{z})
$$

i.e. $\Delta_{0}(\mathrm{z})$ represents the part of $\mathrm{R}_{\mathrm{PT}}(\mathrm{z})$ free of $\mathrm{z}=\mathrm{z}_{0}$ renormalon. As argued above, the $\overline{\mathrm{R}}_{\mathrm{PT}}^{(\mathrm{N})}(\alpha)$ series are convergent. Moreover, the weak coupling expansion of $\Delta_{0}(\alpha)$ follows from eq.(28) by expanding $\Delta_{0}(\mathrm{z})$ around $\mathrm{z}=\mathrm{z}_{0}$ (where it is analytic by definition) :

$$
\Delta_{0}(\mathrm{z})=\sum_{\mathrm{n}=0}^{\infty} \frac{\mathrm{d}_{\mathrm{n}}}{\mathrm{n} !}\left(\mathrm{z}-\mathrm{z}_{0}\right)^{\mathrm{n}}
$$

which gives : 


$$
\Delta_{0}(\alpha)=\exp \left(-\mathrm{z}_{0} / \alpha\right) \sum_{n=0}^{\infty} d_{n} \alpha^{n+1}
$$

In the absence of the other renormalons, $\Delta_{0}(\mathrm{z})$ would be an entire function, and the series eq.(30) could have a finite convergence radius. Note that the $d_{n}$ 's are determined from eq.(28) by :

$$
d_{n}=\lim _{N \rightarrow \infty} d_{n}(N)
$$

with

$$
d_{n}(N)=\frac{d^{n} R_{P T}^{(N)}}{d z^{n}}\left(z=z_{0}\right)+\frac{d^{n} \widetilde{G}_{0}^{(N)}}{d z^{n}}\left(z=z_{0}\right)
$$

6. The extension of the previous method to the case $\delta<0(\gamma>1)$ is straightforward : it suffices to extract the appropriate power of the coupling. For instance, assuming $-1<\delta<0(1<\gamma<2)$, one can apply the previous arguments to the function $\alpha \mathrm{R}$, since $\widetilde{\mathrm{G}}_{0}(\alpha)$ satisfies the modified Borel representation :

$$
\widetilde{G}_{0}(\alpha)=\frac{1}{\alpha} \int_{z_{0}}^{\infty} \mathrm{dz} \exp (-z / \alpha) \widetilde{G}_{0, \text { new }}(\mathrm{z})
$$

with

$$
\frac{\mathrm{d} \widetilde{\mathrm{G}}_{0, \text { new }}}{\mathrm{dz}}=\widetilde{\mathrm{G}}_{0}(\mathrm{z})
$$

which gives :

$$
\widetilde{\mathrm{G}}_{0, \text { new }}(\mathrm{z})=\widetilde{\mathbf{C}}\left(\frac{\mu^{2}}{\mathrm{Q}^{2}}\right)^{\mathrm{d} / 2} \frac{\mathrm{z}_{0}^{\delta}}{\Gamma(\delta+1)} \frac{1}{\left(1-\frac{\mathrm{z}}{\mathrm{z}_{0}}\right)^{-\delta}}\left[1+\mathrm{O}\left(1-\frac{\mathrm{z}}{\mathrm{z}_{0}}\right)\right]
$$

i.e. an integrable singularity. Similarly, consider :

$$
\mathrm{R}_{\mathrm{PT}}(\alpha)=\frac{1}{\alpha} \int_{0}^{\infty} \mathrm{dz} \exp (-\mathrm{z} / \alpha) \mathrm{R}_{\mathrm{PT}, \text { new }}(\mathrm{z})
$$

with

$$
\frac{\mathrm{dR}_{\mathrm{PT}, \text { new }}}{\mathrm{dz}}=\mathrm{R}_{\mathrm{PT}}(\mathrm{z})
$$

We have the new convergent series : 


$$
\begin{aligned}
\mathrm{R}_{\mathrm{PT}, \text { new }}^{\mathrm{reg}(\mathrm{N})}(\alpha) & =\frac{1}{\alpha}\left[\int_{0}^{\infty} \mathrm{dz} \exp (-\mathrm{z} / \alpha) \mathrm{R}_{\mathrm{PT}, \text { new }}^{(\mathrm{N}+1)}(\mathrm{z})+\int_{\mathrm{z}_{0}}^{\infty} \mathrm{dz} \exp (-\mathrm{z} / \alpha) \widetilde{\mathrm{G}}_{0, \text { new }}^{(\mathrm{N}+1)}(\mathrm{z})\right] \\
& =\sum_{\mathrm{n}=0}^{\mathrm{N}} \mathrm{r}_{\mathrm{n}} \alpha^{\mathrm{n}+1}+\exp \left(-\mathrm{z}_{0} / \alpha\right) \sum_{\mathrm{n}=0}^{\mathrm{N}+1} \frac{\mathrm{d}^{\mathrm{n}} \widetilde{\mathrm{G}}_{0, \text { new }}^{(\mathrm{N}+1)}}{\mathrm{dz^{ \textrm {n } }}}\left(\mathrm{z}=\mathrm{z}_{0}\right) \times \alpha^{\mathrm{n}} \\
& \equiv \mathrm{R}_{\mathrm{PT}}^{(\mathrm{N})}(\alpha)+\widetilde{\mathrm{G}}_{0, \text { new }}^{(\mathrm{N})}(\alpha)
\end{aligned}
$$

Note that $\widetilde{\mathrm{G}}_{0, \text { new }}^{(\mathrm{N})}(\alpha)$ is $\mathrm{O}\left(\exp \left(-\mathrm{z}_{0} / \alpha\right)\right)$ this time. In fact we have :

$$
\widetilde{\mathrm{G}}_{0, \text { new }}^{(\mathrm{N})}(\alpha)=\widetilde{\mathrm{G}}_{0, \text { new }}^{(\mathrm{N}+1)}\left(\mathrm{z}=\mathrm{z}_{0}\right) \exp \left(-\mathrm{z}_{0} / \alpha\right)+\widetilde{\mathrm{G}}_{0}^{(\mathrm{N})}(\alpha)
$$

Similarly, if $-2<\delta<-1(2<\gamma<3)$, consider $\alpha^{2} \mathrm{R}$, etc... For arbitrary $\delta<0$, one thus gets a corresponding $\widetilde{\mathrm{G}}_{0 \text {,new }}^{(\mathrm{N})}(\alpha)$ which behaves as $\mathrm{O}\left[\alpha^{-\mathrm{E}(-\delta)} \exp \left(-\mathrm{z}_{0} / \alpha\right)\right]$, where $\mathrm{E}(-\delta)$ is the entire part of $-\delta$. If $\mathrm{E}(-\delta)$ is not very different from $-\delta$, the $\widetilde{\mathrm{G}}_{0 \text {,new }}^{(\mathbb{N})}(\alpha)$ corrections may again be merged with $\mathrm{G}_{0}(\alpha)$ into an "effective condensate" contribution (for the gluon condensate, one gets $-\delta=-2 \beta_{1} / \beta_{0}^{2}=1.6,1.5$ and 1.3 for 3,4 , and 5 flavors respectively).

7. The previous procedure can be easily extended to deal with the non-leading IR renormalons. Consider the next IR singularity at $\mathrm{z}=\mathrm{z}_{1}>\mathrm{z}_{0}$. The OPE, including the next condensate contribution of dimension $d_{1}>d\left(\right.$ with $\left.z_{1}=-d_{1} / 2 \beta_{0}\right)$ yields :

$$
R(Q)=R_{P T}(\alpha)+\left[\widetilde{G}_{0}(\alpha)+G_{0}(\alpha)\right]+\left[\widetilde{G}_{1}(\alpha)+G_{1}(\alpha)\right]+\ldots
$$

with $\widetilde{G}_{1}(\alpha), G_{1}(\alpha)$ given by similar expressions to eq.(11), (12), e.g. :

$$
\widetilde{\mathrm{G}}_{1}(\alpha)=\int_{\mathrm{z}_{1}}^{\infty} \mathrm{dz} \exp (-\mathrm{z} / \alpha) \widetilde{\mathrm{G}}_{1}(\mathrm{z})=\widetilde{\mathrm{C}}_{1}\left(\frac{\mu^{2}}{\mathrm{Q}^{2}}\right)^{\mathrm{d}_{1} / 2} \exp \left(\mathrm{d}_{1} / 2 \beta_{0} \alpha\right)(-\alpha)^{\delta_{1}}[1+\mathrm{O}(\alpha)]
$$

etc... One proceeds by successive steps to "regularize" the $\mathrm{z}=\mathrm{z}_{0}, \mathrm{z}_{1}$, etc... renormalons. First taking care of the $\mathrm{z}=\mathrm{z}_{0}$ renormalon as above, one gets (see eq.(27)) :

$$
R(Q)=\bar{R}_{\mathrm{PT}}(\alpha)+\left[\Delta_{0}(\alpha)+\mathrm{G}_{0}(\alpha)\right]+\widetilde{\mathrm{G}}_{1}(\alpha)+\mathrm{G}_{1}(\alpha)+\ldots
$$

where $\widetilde{\mathrm{G}}_{1}(\alpha)$ must "regularize" the $z=z_{1}$ renormalon expected to occur in $\Delta_{0}(\alpha)+\mathrm{G}_{0}(\alpha)$. This means, at the Borel transform level, that : 


$$
\Delta_{1}(\mathrm{z})=\left[\Delta_{0}(\mathrm{z})+\mathrm{G}_{0}(\mathrm{z})\right]+\widetilde{\mathrm{G}}_{1}(\mathrm{z})
$$

must be free of $z=z_{1}$ renormalon, and one can write, similarly to eq.(27) :

$$
\left[\Delta_{0}(\alpha)+G_{0}(\alpha)\right]+\widetilde{G}_{1}(\alpha)=\bar{\Delta}_{0}(\alpha)+\bar{G}_{0}(\alpha)+\Delta_{1}(\alpha)
$$

where

$$
\begin{aligned}
& \bar{\Delta}_{0}(\alpha)=\int_{\mathrm{z}_{0}}^{\mathrm{z}_{1}} \mathrm{dz} \exp (-\mathrm{z} / \alpha) \Delta_{0}(\mathrm{z}) \\
& \overline{\mathrm{G}}_{0}(\alpha)=\int_{\mathrm{z}_{0}}^{\mathrm{z}_{1}} \mathrm{dz} \exp (-\mathrm{z} / \alpha) \mathrm{G}_{0}(\mathrm{z}) \\
& \Delta_{1}(\alpha)=\int_{\mathrm{z}_{1}}^{\infty} \mathrm{dz} \exp (-\mathrm{z} / \alpha) \Delta_{1}(\mathrm{z})
\end{aligned}
$$

The total $\mathrm{R}$ then appears in the fully regularized form :

$$
\mathrm{R}(\mathrm{Q})=\overline{\mathrm{R}}_{\mathrm{PT}}(\alpha)+\left[\overline{\mathrm{G}}_{0}(\alpha)+\bar{\Delta}_{0}(\alpha)\right]+\left[\mathrm{G}_{1}(\alpha)+\Delta_{1}(\alpha)\right]+\ldots
$$

(regularizing the $\mathrm{z}=\mathrm{z}_{2}$ renormalon will turn $\mathrm{G}_{1}(\alpha)+\Delta_{1}(\alpha)$ into $\overline{\mathrm{G}}_{1}(\alpha)+\bar{\Delta}_{1}(\alpha)=$ $\int_{z_{1}}^{z_{2}} d z \exp (-z / \alpha)\left(G_{1}(z)+\Delta_{1}(z)\right)$, etc...). Define :

and

$$
\Delta_{0}^{(N)}(\mathrm{z})=\sum_{n=0}^{N} \frac{d_{n}}{n !}\left(z-z_{0}\right)^{n}
$$

$$
\Delta_{1}^{(N)}(z)=\sum_{n=0}^{N} \frac{d_{n, 1}}{n !}\left(z-z_{1}\right)^{n}
$$

and call $\mathrm{G}_{0}^{(\mathrm{N})}(\mathrm{z})$ the expansion eq.(12) trunkated at order $\mathrm{N}$. One can then recast eq.(43) into the more standard looking form : 


$$
\begin{aligned}
\mathrm{R}(\mathrm{Q}) \sim \mathrm{R}^{\left(\mathrm{N}^{\prime}\right)}(\alpha) & =\int_{0}^{\infty} \mathrm{dz} \exp (-\mathrm{z} / \alpha) \mathrm{R}_{\mathrm{PT}}^{(\mathrm{N})}(\mathrm{z})+\int_{\mathrm{z}_{0}}^{\infty} \mathrm{dz} \exp (-\mathrm{z} / \alpha) \widetilde{\mathrm{G}}_{0}^{\left(\mathrm{N}^{\prime}\right)}(\mathrm{z}) \\
& +\int_{\mathrm{z}_{0}}^{\infty} \mathrm{dz} \exp (-\mathrm{z} / \alpha) \mathrm{G}_{0}^{(\mathrm{N})}(\mathrm{z})+\int_{\mathrm{z}_{1}}^{\infty} \mathrm{dz} \exp (-\mathrm{z} / \alpha) \widetilde{\mathrm{G}}_{1}^{\left(\mathrm{N}^{\prime}\right)}(\mathrm{z}) \\
& +\int_{\mathrm{z}_{1}}^{\infty} \mathrm{dz} \exp (-\mathrm{z} / \alpha) \mathrm{G}_{1}(\mathrm{z})+\ldots \\
& \equiv \mathrm{R}_{\mathrm{PT}}^{(\mathrm{N})}(\alpha)+\left[\widetilde{\mathrm{G}}_{0}^{\left(\mathrm{N}^{\prime}\right)}(\alpha)+\mathrm{G}_{0}^{(\mathrm{N})}(\alpha)\right]+\left[\widetilde{\mathrm{G}}_{1}^{\left(\mathrm{CN}^{\prime}\right)}(\alpha)+\mathrm{G}_{1}(\alpha)\right]+\ldots
\end{aligned}
$$

with (see eq.(28)) :

$$
\widetilde{\mathrm{G}}_{0}^{(' \mathrm{~N})}(\mathrm{z})=\Delta_{0}^{(\mathrm{N})}(\mathrm{z})-\mathrm{R}_{\mathrm{PT}}^{(\mathrm{N})}(\mathrm{z})
$$

and (see eq.(40)) :

$$
\widetilde{\mathrm{G}}_{1}^{\left(\mathrm{N}^{\prime}\right)}(\mathrm{z})=\Delta_{1}^{(\mathrm{N})}(\mathrm{z})-\left[\Delta_{0}^{(\mathrm{N})}(\mathrm{z})+\mathrm{G}_{0}^{(\mathrm{N})}(\mathrm{z})\right]
$$

where the " ' are put as a reminder that the functions on the right hand side of eq.(46) are not all expanded around the same value of $z$. Note also the $d_{n, 1}$ coefficients in eq.(44) are to be considered as free parameters : they cannot be computed in term of the renormalon residue (at the difference of the $d_{n}$ 's), because of complications $\left(\widetilde{G}_{1}(z)\right.$ is presumably singular also at $\left.z=z_{0}\right)$ arising from the expected presence of $z=z_{1}$ renormalon in $G_{0}(z)$. For the same reason, one cannot think of $\widetilde{G}_{1}(\alpha)$ as the "perturbation theory determined part" of the $\mathrm{G}_{1}$ condensate.

\section{References}

[1] M.A. Shifman, A.I.Vainshtein and V.I. Zakharov, Nucl. Phys. B147 (1979) 385, 448, 519.

[2] F. David, Nucl. Phys. B234 (1984) 237 ; B263 (1986) 637.

[3] G. 't Hooft, in "The Whys of Subnuclear Physics", Erice 1977, ed. A. Zichichi (Plenum, New York 1977). 
[4] E.B. Bogomolny and V.A. Fateyev, Phys. Lett. B71 (1977) 93.

[5] G. Parisi, Phys. Lett. B76 (1978) 65 ; Nucl. Phys. B150 (1979) 163.

[6] A.H. Mueller, Nucl. Phys. B250 (1985) 327 ; in "QCD-20 Years Later", ed. P.M. Zerwas and H.A. Kastrup (World Scientific, Singapore 1993).

[7] G. Grunberg, Phys. Lett. B304 (1993) 183 ; Ecole Polytechnique preprint A265.1093 (contribution to "Quantum Field Theoretic Aspects of High Energy Physics", Kyffhäuser, Germany, september 1993), to be published.

[8] M. Beneke, Phys. Lett. B307 (1993) 154.

[9] V.I. Zakharov, Nucl. Phys. B385 (1992) 452 ; M. Beneke and V.I. Zakharov, Phys. Lett. B312 (1993) 340.

[10] A.H. Mueller, Phys. Lett. B308 (1993) 355.

[11] see e.g. J.C. Le Guillou and J. Zinn-Justin, eds., Large Order Behavior of Perturbation Theory, Current Physics Sources and Comments Vol. 7, North-Holland (1990). 